\title{
Diabetes Melito Neonatal
}

\section{RESUMO}

O diabetes neonatal (DN) é uma condição rara caracterizada por hiperglicemia, que necessita de tratamento com insulina, diagnosticado nos primeiros meses de vida. Clinicamente pode ser classificado em DN transitório quando ocorre remissão da doença em poucos meses, podendo haver recorrência posterior; ou permanente quando, como o nome indica, não ocorre remissão. Ambas as condições são geneticamente heterogêneas; entretanto a maioria dos casos de DN transitório é decorrente de anormalidades da região de imprinted no cromossomo 6q24. Mutações ativadoras em heterozigose no gene KCNJ11, que codifica a subunidade Kir6.2 do canal de potássio ATP-sensível, são a causa mais comum de DN permanente. No presente artigo, discutimos as características clínicas do DN, os mecanismos moleculares envolvidos e suas implicações terapêuticas. (Arq Bras Endocrinol Metab 2008;52/2:181-187)

Descritores: Diabetes neonatal; Genética; Terapêutica

\section{ABSTRACT}

\section{Neonatal Diabetes Mellitus.}

Neonatal diabetes is a rare condition characterized by hyperglycemia, requiring insulin treatment, diagnosed within the first months of life. The disorder may be either transient, resolving in infancy or early childhood with possible relapse later, or permanent in which case lifelong treatment is necessary. Both conditions are genetically heterogeneous; however, the majority of the cases of transient neonatal diabetes are due to abnormalities of an imprinted region of chromosome 6q24. For permanent neonatal diabetes, the most common causes are heterozygous activating mutations of $K C N J 11$, the gene encoding the Kir6.2 subunit of the ATP-sensitive potassium channel. In this article we discuss the clinical features of neonatal diabetes, the underlying genetic defects and the therapeutic implications. (Arq Bras Endocrinol Metab 2008;52/2:181-187)

Keywords: Neonatal diabetes; Genetic; Therapeutic

\section{INTRODUÇÃO}

\footnotetext{
PESAR DO PERÍODO NEONATAL corresponder ao período do nascimento até o $28^{\circ}$ - dia de vida, o diabetes neonatal (DN) tem sido definido na literatura como a presença de hiperglicemia, que necessite de tratamento com insulina, nos três primeiros meses de vida $(1,2)$. Entretanto, como sugerido por Shield (3), em termos de idade, um ponto de corte aos 6 meses parece mais apropriado, uma vez que possivelmente todos os casos de diabetes melito diagnosticados antes dessa idade são devidos a mutações em um único gene, e após os 6 meses de idade diabetes melito tipo 1 auto-imune representa a causa mais comum da doença (4).
}

revisão

LUCIMARY C. GURGEL

Regina S. MoIsÉs

Disciplina de Endocrinologia, Escola Paulista de Medicina, Universidade Federal de São Paulo, SP, Brasil.
Recebido em 11/1/2008

Aceito em 21/1/2008 
O DN é uma condição rara, com incidência estimada de 1 em 400.000 a 500.000 nascidos vivos $(5,6)$. Em aproximadamente metade dos casos, o DN é transitório (DNT) entrando em remissão em média dentro de três meses e podendo recidivar durante a infầncia ou a adolescência, e na outra metade dos casos, o DN é permanente (DNP). Não existem características clínicas que possam predizer se um neonato com diabetes melito apresenta a forma transitória ou permanente.

\section{CARACTERÍSTICAS CLÍNICAS}

\section{Diabetes neonatal transitório}

O DNT representa cerca de $50 \%$ a $60 \%$ dos casos de DN (6-8). Os indivíduos afetados desenvolvem hiperglicemia com hipoinsulinemia precocemente, com duração de necessidade de insulina exógena de 4 a 60 semanas, período após o qual entram em remissão (9). Em uma grande casuística de pacientes ingleses com DNT, Temple e col. observaram a mediana de idade de apresentação de três dias, com intervalo de poucas horas de vida até 31 dias (9). Entretanto, após período variável de remissão, com resposta insulínica normal à sobrecarga intravenosa de glicose (10), hiperglicemia permanente desenvolveu-se durante a fase de adolescência ou em adulto jovem em grande proporção desses indivíduos. Em um estudo francês, DNP ocorreu em cinco de sete pacientes com DNT após os 8 anos de idade (11); similarmente entre pacientes ingleses verificou-se a recorrência do diabetes melito em 11 de 18 pacientes maiores de 4 anos de idade (9). Esses dados enfatizam a necessidade de seguimento prolongado nesses pacientes.

Além da hiperglicemia, o DNT é caracterizado por retardo no crescimento intra-uterino $(6,9,11)$ refletindo o papel importante da insulina no crescimento fetal, especialmente durante o último trimestre da gestação. Ainda Temple e col. observaram macroglossia em 23\% dos portadores de DNT e hérnia umbelical em 7\% (9). Em comparação com os portadores de DNP, os pacientes com DNT apresentam hiperglicemia em idade mais precoce, menor peso por ocasião do diagnóstico, necessitam de doses menores de insulina para o controle metabólico e apresentam menor freqüência de cetoacidose (11). Entretanto, existe considerável sobreposição das manifestações clínicas entre os dois grupos, não permitindo, por ocasião do diagnóstico, inferências se o diabetes será transitório ou permanente.
Anticorpos antiilhotas foram negativos nos pacientes testados $(6,9,11)$, indicando uma etiologia não-auto-imune na gênese do diabetes.

Os mecanismos que levam à falência das células- $\beta$ no período neonatal, seguido de recuperação na infância e recorrência na adolescência permanecem incertos, assim como a contribuição relativa da redução do número de células- $\beta$ e diminuição da função dessas células nesse processo. Ma e col. desenvolveram uma linhagem de camundongos transgênicos que superexpressam o lócus do DNT humano (12). Verificou-se que intraútero esses animais apresentavam redução da massa de células- $\beta$. Posteriormente, no período neonatal ocorreu compensação com aumento do número de células- $\beta$, entretanto o conteúdo de insulina é menor que nos animais-controle. Uma compensação plena ocorre então nos animais jovens por meio do aumento substancial do número das células- $\beta$; entretanto esse aumento compensatório não é mantido, ocorrendo a intolerância à glicose na fase adulta. Esses dados indicam que alterações no desenvolvimento pancreático e diminuição da função das células- $\beta$ estão envolvidas na patogênese da doença.

\section{Diabetes neonatal permanente}

O DNP é aquele que ocorre nos primeiros meses de vida e, como o nome indica, não entra em remissão. É menos comum que a forma transitória (8).

No DNP, a idade ao diagnóstico é mais tardia que no DNT, ocorrendo com cerca de 27 dias (intervalo de l a 127 dias) (11). Retardo do crescimento intra-uterino foi observado em $36 \%$ dos casos, e no DNT esteve presente em $74 \%$ dos afetados. Essas diferenças sugerem que as formas de DNP e DNT tenham mecanismos diferentes, um defeito no desenvolvimento e/ou função das células- $\beta$ pode estar presente nos períodos fetal e pós-natal precoce no DNT, enquanto uma falência das células- $\beta$ após o nascimento ocorreria no DNP (11).

O diabetes melito diagnosticado antes dos 6 meses de idade é raramente causado por processo auto-imune. Iafusco e col. demonstraram que em crianças com diabetes diagnosticado antes dos 6 meses, 76\% apresentavam um genótipo HLA "protetor" para o diabetes melito tipo 1 e também menor freqüência de marcadores auto-imunidade quando comparadas com crianças diagnosticadas após os 6 meses de idade (4). Da mesma forma, Edghill e col. verificaram que em indivíduos 
com diagnóstico antes dos 6 meses de idade, a freqüência dos genótipos HLA de risco para diabetes melito tipo 1 foi similar ao da população controle (13). Esses dados indicam que o diabetes melito diagnosticado antes dos 6 meses de idade difere do diabetes diagnosticado em idades mais tardias, e a maioria desses casos não tem uma etiologia auto-imune.

\section{BASES MOLECULARES DO DN}

\section{Anomalias no braço longo do cromossomo 6}

A maioria (cerca de 70\%) dos pacientes com DNT apresenta anormalidades no cromossomo 6q24. Três tipos de anormalidades foram verificados: dissomia uniparental paterna do cromossomo 6 (UPD6), ou seja, herança de duas cópias do mesmo cromossomo 6 do pai sem a contribuição materna; duplicação de herança paterna do braço longo do cromossomo 6 e defeitos de metilação (14-18). Apenas se a duplicação for de herança paterna é que ocorre o DNT. As anomalias no cromossomo 6 são devidas a alterações de imprinting. Em termos simples, imprinting consiste da supressão de certos genes através da adição de grupos metil, geralmente na região promotora, prevenindo a transcrição gênica. Portanto, duas cópias do cromossomo paterno 6 , uma duplicação paterna do 6q24 ou perda de imprinting (perda de metilação) do 6q24 materno levam à superexpressão do alelo paterno causando o DNT. Dois genes localizados nessa região são candidatos para a doença: o gene que codifica o fator de crescimento $Z A C$ que regula o ciclo celular e a apoptose e o gene HYMAI de função desconhecida (19). Em um modelo animal que superexpressa o lócus DNT humano verificou-se redução na expressão do fator de transcrição IPF-1 no pâncreas desses camundongos (12). O IPF- 1 é um gene envolvido no controle do desenvolvimento pancreático, sendo responsável pelo desenvolvimento coordenado do pâncreas intra-útero e também pela integridade funcional das células- $\beta$ pancreáticas. Não se verificaram diferenças fenotípicas entre os pacientes com UPD6, duplicação do 6q24, defeitos de metilação ou sem anomalias identificadas no cromossomo $6(9)$.

\section{Mutações no Canal de Potássio ATP-sensível $\left(\mathrm{K}^{+}{ }_{\text {ATP }}\right)$}

Os canais $\mathrm{K}_{\text {ATP }}^{+}$são um complexo octamérico composto por quatro subunidades Kir6.2 (inwardly rectifying potassium channels) que formam o poro do canal e quatro subunidades regulatórias SURI (sulphonylurea receptor) presentes nas células- $\beta$ pancreáticas. A subunidade Kir6.2 é codificada pelo gene $K C N J 11$ e a subunidade SURl pelo gene $A B C C 8$, ambas localizadas no cromossomo 11 (lócus llp 15.1). Esses canais têm papel importante na secreção de insulina fazendo a ligação entre o metabolismo celular e a atividade elétrica da membrana plasmática, sendo tanto o Kir6.2 quanto SURI vitais para a regulação adequada da secreção de insulina. A glicose entra na célula- $\beta$ através da proteína transportadora GLUT-2, sendo então metabolizada por enzimas da via glicolítica, incluindo a glicoquinase, para produzir ATP. O aumento da relação ATP/ADP intracelular leva ao fechamento do canal $\mathrm{K}^{+}{ }_{\mathrm{ATP}}$ e à despolarização da membrana plasmática. O canal de cálcio voltagem-sensível então se abre e o influxo de cálcio resulta exocitose dos grânulos de insulina.

\section{Mutações no gene KCNJ11}

Em abril de 2004, Gloyn e col. descreveram pela primeira vez as mutações ativadoras no gene que codifica a subunidade Kir6.2 como causa de DNP (2). Desde então, vários estudos reportaram mutações nesse gene e verificou-se ser essa a causa mais comum de DNP, ocorrendo em $31 \%$ a $64 \%$ dos casos (20-22). Mutações funcionalmente menos graves resultam DNT em cerca de $10 \%$ dos casos. A maioria dos indivíduos afetados não apresenta história familiar, uma vez que em $90 \%$ dos casos as mutações são espontâneas, ocorrendo de novo.

As mutações ativadoras no gene KCNJII provocam falência no fechamento do $\mathrm{K}^{+}$ATP na presença de ATP. Isso resulta grande influxo de potássio mantendo a membrana plasmática hiperpolarizada, prevenindo assim a secreção de insulina (23).

Nos pacientes afetados, em $75 \%$ dos casos, o diagnóstico de diabetes melito é feito antes dos 3 meses de idade, em média com 7 semanas (2) e apresentam baixo peso ao nascimento refletindo hipoinsulinemia intraútero. Níveis de peptídeo C são indetectáveis mesmo após estímulo com glucagon $(2,21)$ e $30 \%$ dos casos apresentam-se em cetoacidose (24). Além do diabetes melito, alterações neurológicas estão presentes em cerca de $20 \%$ dos pacientes. A forma mais grave consiste de retardo no desenvolvimento, fraqueza muscular e epilepsia, tendo sido proposto o nome de síndrome DEND (developmental delay, epilepsy and neonatal diabetes) (25). Uma forma mais branda de DN consiste de retardo no desenvolvimento e/ou fraqueza muscular e ausência de epilepsia, sendo referida síndrome DEND interme- 
diária. Os sintomas neurológicos verificados na síndrome DEND são provavelmente decorrentes do aumento da atividade dos canais $\mathrm{K}^{+}{ }_{\text {ATP }}$ em outros tecidos além das células- $\beta$, tais como músculo e/ou nervos (26).

Existem evidências para a relação fenótipo-genótipo nas mutações do gene $K C N J 11$. Apesar de não ser absoluto, em geral mutações associadas apenas com DN (permanente ou transitório), localizam-se na região do sítio de ligação do ATP, enquanto mutações que causam alterações neurológicas ocorrem em resíduos mais distantes do sítio de ligação do ATP, sendo principalmente dentro da porção helicoidal (26).

\section{Mutações no gene ABCC8}

Como as mutações inativadoras nos genes que codificam o Kir6.2 e SURl são causa de hipoglicemia hiperinsulinemica (27), e mutações ativadoras no Kir6.2 causam DN, Proks e col. levantaram a hipótese que mutações ativadoras no SURI também seriam causa de DN. De fato, esses pesquisadores identificaram uma mutação ativadora no gene $A B C C 8 \mathrm{em}$ um paciente com síndrome DEND (28). Posteriormente, Babenko e col. realizaram o rastreamento de mutações nesse gene em pacientes com DNP ou DNT e em 12\% dos casos mutações foram identificadas, sendo a maioria em DNT (29). Portanto, mutações no gene $K C N J 11$ são na maioria das vezes associadas com DNP, enquanto mutações no gene $A B C C 8$ são mais freqüentemente associadas com DNT. Ainda, verificou-se que alguns pais dos probandos com mutações no gene $A B C C 8 \mathrm{e}$ também portadores da mutação, o diagnóstico do diabetes melito foi feito na idade adulta, ou diabetes nem sempre esteve presente nesse adultos carreadores $(29,30)$. Esses dados levaram à proposição de que mutações no gene $A B C C 8$ resultam forma monogênica de diabetes melito, não apenas no período neonatal mas com idade de início e penetrância variáveis.

\section{Mutações no gene da insulina}

Recentemente, identificaram-se mutações no gene da insulina como causa de DN (31). Há descrições da década de 1980 de mutações no gene da insulina que afetam a atividade biológica da insulina, mas não alteram significantemente sua biossíntese. $\mathrm{O}$ desenvolvimento de diabetes melito, nesses casos, não foi uniforme, ocorrendo apenas na presença de resistência à insulina $\mathrm{e}$ na idade adulta, sendo muitos carreadores assintomáticos, porém com hiperinsulinemia (32-34). Diferente- mente, as mutações identificadas em pacientes com DN são mais graves resultando diabetes em idade precoce. Em um grande estudo colaborativo internacional, identificaram-se mutações no gene da insulina em $12 \%$ dos pacientes com DNP, sendo a segunda causa mais comum, após mutações no gene KCNJII, de DNP (35). Os indivíduos afetados apresentaram idade média ao diagnóstico do diabetes melito de nove semanas (> 90\% antes dos 6 meses), em cetoacidose ou hiperglicemia importante (mediana da glicemia ao diagnóstico: 681 $\mathrm{mg} / \mathrm{dl}$ ) e níveis de peptídeo C indetectáveis ou muito baixos. Verificou-se também baixo peso ao nascimento indicando secreção reduzida de insulina in útero (31).

\section{Mutações no gene da glicoquinase}

A glicoquinase, enzima da via glicolítica, é reguladora do metabolismo da glicose nas células- $\beta$, controlando a secreção de insulina. Mutações em heterozigose no gene da glicoquinase são causa de MODY 2, entretanto quando presentes em homozigose ou heterozigose composta são uma causa bastante rara de DNP. Mutações em homozigose foram descritas inicialmente em dois probandos apresentando-se com DNP no primeiro dia de vida. Os pais apresentavam consangüinidade $\mathrm{e}$ intolerância à glicose, sendo heterozigotos para as mesmas mutações presentes nos filhos (36). Apesar de ser uma condição bastante rara, recomenda-se a pesquisa de mutações no gene da glicoquinase em portadores de DNP em que ambos os pais apresentam intolerância à glicose (3).

\section{Mutações no gene IPF-1 (insulin promoter factor 1)}

O IPF- 1 é um gene envolvido no controle do desenvolvimento pancreático, sendo responsável pelo desenvolvimento coordenado do pâncreas intra-útero e também pela integridade funcional das células- $\beta$ pancreáticas. Mutações em heterozigose causam MODY 4, e mutações em homozigose ou heterozigose composta foram reportadas em portadores de DNP e agenesia pancreática $(37,38)$.

\section{Formas sindrômicas de DN}

Além das condições discutidas anteriormente, há ainda doenças multissistêmicas, bastante raras, que incluem DN. Entre essas síndromes inclui-se a síndrome IPEX (immunodysregulation polyendocrinopathy and enteropathy X-linked syndrome), que é uma desordem fatal, 
bastante rara, de herança ligada ao cromossomo X e caracterizada por diarréia intratável com atrofia das vilosidades intestinais, eczema, anemia hemolítica e diabetes melito de etiologia auto-imune e hipotireoidismo. Essa síndrome está associada com mutações no gene FOXP3 que codifica uma proteína denominada scurfina, importante para a homeostase imune normal (39). A síndrome de Wolcott-Rallison é uma alteração de herança recessiva autossômica recessiva caracterizada por diabetes melito de início na infância (freqüentemente no período neonatal), displasia espôndilo-epifisária, hepatomegalia e insuficiência renal. Está associada com mutações no gene EIF2AK3 (40). Ainda, diabetes neonatal com hipoplasia pancreática e cerebelar, de herança autossômica recessiva, foi associada com mutações no gene PTFIA (41). Esse gene está envolvido no desenvolvimento pancreático, sendo também expresso no cerebelo.

\section{IMPLICAÇÕES TERAPÊUTICAS}

Uma vez que as sulfonilureas, classe de drogas utilizadas no tratamento do diabetes melito tipo 2, causam o fechamento dos canais de $\mathrm{K}^{+}{ }_{\text {ATP }}$ por um mecanismo independente do ATP, sugere-se que essa droga possa também ser utilizada no DN causado por mutações nos canais de $\mathrm{K}_{\text {ATP. }}^{+}$Em três pacientes com mutações no gene KCNJ11 que apresentavam secreção de insulina mínima em resposta à glicose endovenosa, Gloyn e col. observaram secreção substancial de insulina em resposta à tolbutamida (2). Subseqüentemente, reportagens demonstraram a transferência bem-sucedida de insulina para sulfonilurea em muitos dos pacientes portadores de mutações no gene $K C N J 11(21,42,43)$. Em um estudo colaborativo europeu foi possível a substituição de insulina por sulfonilurea em $90 \%$ dos pacientes e em todos houve melhora no controle metabólico. Entre os pacientes em que não foi possível a suspensão da insulina, 80\% apresentavam alterações neurológicas, em contraste com apenas $14 \%$ do grupo em que a transferência foi possível (44). As doses necessárias de sulfonilurea nesses pacientes são mais elevadas que as usualmente utilizadas para o tratamento do diabetes melito tipo 2 . Pessoalmente, tivemos a gratificante experiência da transferência de insulina por glibenclamida em um paciente com síndrome DEND intermediária em razão da mutação G53D no gene KCNJll que vinha em uso de insulina há 26 anos (45). Após a transferência houve melhora importante no controle metabólico e de algumas funções neurológicas e desaparecimento dos episódios de hipoglicemia seguidos de crises convulsivas. Resultados similares foram obtidos em pacientes com mutações ativadoras no SURl $(20,30)$. Portanto, tratamento com sulfonilurea parece ser seguro, mais eficiente que insulina e deve reduzir o risco de complicações crônicas do diabetes em conseqüência de melhor controle metabólico em portadores de DN causado por mutações ativadoras no canal de $\mathrm{K}^{+}{ }_{\text {ATP }}$ (46). Entretanto, as demais causas de DN devem ser tratadas com insulina.

\section{CONSIDERAÇÕES FINAIS}

Apesar do DN ser uma condição rara, a identificação dos defeitos genéticos envolvidos leva ao melhor entendimento das disfunções da célula- $\beta$, contribuindo assim com as formas mais comuns da doença. Possivelmente os mecanismos moleculares envolvidos no DN também apresentem relevância no diabetes melito tipo 2 . Ainda, os recentes avanços obtidos na identificação dos mecanismos moleculares do DN demonstram como o conhecimento exato da fisiopatologia pode ter importantes implicações no tratamento desses pacientes.

\section{REFERÊNCIAS}

1. Hattersley AT, Pearson ER. Pharmacogenetics and beyond: the interaction of the interaction of therapeutic response, B-cell physiology and genetics in diabetes. Endocrinology. 2006; 143:2657-63.

2. Gloyn AL, Pearson ER, Anticliff JF, Proks P, Bruining GJ, Slingerland AS, et al. Activating muttions in the gene encoding the ATP-sensitive potassium-channel subunit Kir6.2 and permanent neonatal diabetes. N Engl J Med. 2004;350:1838-49.

3. Shield JPH. Neonatal diabetes: how research unraveling the genetic puzzle has both widened our understanding of pancreatic development whilst improving children's quality of life. Horm Res. 2007;67:77-83.

4. Iafusco D, Stazi MA, Cotichini R, Cotellessa M, Martinucci ME, Mazzella M, et al., and the early onset diabetes study group of the Italian Society of Pediatric Endocrinology and Diabetology. Permanent diabetes in the first year of life. Diabetologia. 2002;45:798-804.

5. von Muhlendahl KE, Herkenhoff $\mathrm{H}$. Long-term course of neonatal diabetes. N Engl J Med. 1995;333:704-8.

6. Shield JPH, Gardner RJ, Wadswoth EJK, Whiteford ML, James RS, Robinson DO, et al. Aetiopathology and genetic basis of neonatal diabetes. Arch Dis Child. 1997;76: F39-42. 
7. Slingerland AS, Hattersley AT. Mutations in the Kir6.2 subunit of the KATP channel and permanent neonatal diabetes: new insights and new treatment. Ann Med. 2005;37:186-95.

8. Polak M, Cavé H. Neonatal diabetes mellitus: a disease linked to multiple mechanisms. Orphanet J Rare Dis Mar. 2007;2:12.

9. Temple IK, Gardner RJ, Mackay DJG, Barber JCK, Robinsons DO, Shield JPH. Transient neonatal diabetes. Widening the understanding of the etiopathogenesis of diabetes. Diabetes. 2000;49:1359-66.

10. Schiff D, Cole E, Stern L. Metabolic and growth patterns in transient neonatal diabetes. N Engl J Med. 1972;287:119-22.

11. Metz C, Cavé H, Bertrand AM, Deffert C, Gueguen-Giroux B, Czernichow $P$, et al, the NDM French Study Group. Neonatal diabetes mellitus: chromosomal analysis in transient and permanent cases. J Pediatr. 2002;141:483-9.

12. Ma D, Shield JPH, Leclerc I, Knauf C, Buecelin R, Rutter GA, et al. Impaired glucose homeostasis in transgenic mice expressing the human transient neonatal diabetes mellitus locus, TNDM. J Clin Invest. 2004;114:339-48.

13. Edghill EL, Dix RJ, Flanaghan SE, Bingley PJ, Hattersley AT, Ellard S, et al. HLA genotyping supports a nonautoimmune etiology in patients diagnosed with diabetes under the age of 6 months. Diabetes. 2006;55:1895-8.

14. Temple IK, James RS, Crolla JA, Sitch FL, Jacobs PA, Howell WM, et al. An imprinted gene(s) for diabetes? Nat Genet. 1995;9:110-2.

15. Whiteford ML, Narendra A, White MP, Cooke A, Wilkinson AG, Robertson KJ, et al. Paternal uniparental disomy for chromosome 6 causes transient neonatal diabetes. J Med Genet. 1997;34:67-168.

16. Pivinick EK, Qumisiyech MB, Tharapel AT, Summitt JB, Wilroy RS. Partial duplication of the long arm of chromosome 6: a clinically recognizable syndrome. J Med Genet. 1990;27:523-6.

17. Gardner RJ, Mackay DJ, Mungall AJ, Polychronakos C, Siebert $\mathrm{R}$, Shield JP, et al. An imprinted locus associated with transient neonatal diabetes mellitus. Hum Mol Genet. 2000;9:589-96.

18. Temple IK, Gardner RJ, Robinson DO, Kibirige MS, Fergusson AW, Baum JD, et al. Further evidence for an imprinted gene for neonatal diabetes localized to chromosome 6q22-q23. Hum Mol Genet. 1996;5:1117-21.

19. Arima T, Drewell RA, Arney KL, Inoue J, Makita Y, Hata A, et al. A conserved imprinting control region at the HYMAI/ZAC domain is implicated in transient neonatal diabetes mellitus. Hum Mol Genet. 2001;10:1475-83.

20. Massa O, lafusco D, D'Amato E, Gloyn AL, Hattersley AT, Pasquino $B$, et al., Barbetti $F$ and Early Onset Diabetes Study Group of the Italian Society of Pediatric Endocrinology and Diabetology. KCNJ11 activating mutations in Italian patients with permanent neonatal diabetes. Hum Mutat. 2005;25:22-7.

21. Sagen JV, Raeder H, Hathoud E, Shehadeh N, Gudmundsson $\mathrm{K}$, Baevre $\mathrm{H}$ et al. Permanent neonatal diabetes due to mutations in the KCNJ11 encoding Kir6.2. Patient characteristics and initial response to sulphonylurea therapy. Diabetes. 2004:53:2713-8.

22. Vaxilaire M, Populaire $C$, Busiah $K$, Cavé H, Gloyn AL, Hattersley AT, et al. Kir6.2 mutations are a common cause of permanent neonatal diabetes in a large cohort of French patients. Diabetes. 2004;53:2719-22.

23. Hattersley AT. Molecular genetics goes to the diabetes clinic. Clin Med. 2005;5:476-81.
24. Slingerland AS, Hattersley AT. Mutations in the Kir6.2 subunit of the KATP channel and permanent neonatal diabetes: new insights and new treatment. Ann Med. 2005;37:186-95.

25. Proks P, Girard C, Haider S, Gloyn AL, Hattersley AT, Sansom SP, et al. A gating mutation at the internal mouth of the Kir6.2 pore is associated with DEND syndrome. EMBO Rep. 2005;6:470-5.

26. Hattersley AT, Aschroft FM. Activating mutations in the Kir6.2 and neonatal diabetes. New clinical syndromes, new scientific insights, and new therapy. Diabetes. 2005;54:2503-13.

27. Hussain K. Congenital hyperinsulinism. Semin Fetal Neonatal Med. 2005;10:369-76.

28. Proks $P$, Arnold AL, Bruining J, Girard C, Flanagan SE, Larkin $B$, et al. A heterozygous activating mutation in the sulphonylurea receptor SUR1 (ABCC8) causes neonatal diabetes. J Hum Mol Genet. 2006;15:1793-800.

29. Babenko AP, Polak M, Cavé H, Busiah K, Czernichow P, Scharfmann $R$, et al. Activating mutations in the ABCC8 gene in neonatal diabetes mellius. N Engl J Med. 2006;355:456-66.

30. Vaxillaire M, Dechaume A, Busiah K, Cavé H, Pereira S, Scharfmann R, et al., and the SUR1-Neonatal Diabetes Study Group. New ABCC8 mutations in relapsing neonatal diabetes and clinical features. Diabetes. 2007;56:1737-41.

31. Stoy J, Edghill EL, Flanagan SE, Ye Honggang, Paz VP, Pluzhnikov A, et al., for the Neonatal Diabetes International Collaborative Group. Insulin gene mutations as a cause of permanent neonatal diabetes. Proc Natl Acad Sci USA. 2007;18:15040-4.

32. Shoelson $S$, Haneda M, Blix $P$, Nanjo A, Sanke T, Inouye $K$, et al. Three mutant insulins in man. Nature. 1983;302:540-3.

33. Nanjo K, Sanke T, Miyano M, Okai K, Sowa R, Kondo M, et al. Diabetes due to secretion of a structurally abnormal insulin (insulin Wakayama). Clinical and functional characteristics of [LeuA3] insulin. J Clin Invest. 1986;77:514-9.

34. Nanjo K, Kondo M, Sanke T, Nishi M. Abnormal insulinemia. Diabetes Res Clin Pract. 1994;24 suppl:S135-41.

35. Edghill EL, Flanagan SE, Patch AM, Boustred C, Parrish A, Shields B, et al., the Neonatal Diabetes Internacional Collaborative Group, Hattersley AT, Ellard S. Insulin mutation screening in 1044 patients with diabetes: mutations in the INS gene are a common cause of neonatal diabete but a rare cause of diabetes diagnosed in childhood or adulthood. Diabetes. 2007;Dec 27 [Epud ahead of print].

36. Njolstad PR, Sagen JV, Cuesta-Munoz A, Bjorkhaug L, Massa $\mathrm{O}$, Barbetti $\mathrm{F}$, et al. Neonatal diabetes mellitus due to complete glucokinase deficiency. N Engl J Med. 2001;344:1588-92.

37. Stoffers DA, Zinkin NT, Stanojevic V, Clarke WI, Habener JF. Pancreatic agenesis attributable to a single nucleotide deletion in the human IPF1 gene coding sequence. Nat Genet. 1997;15:106-10.

38. Schwitzgebel VM, Mamin A, Brun T, Ritz-Laser B, Zaiko M, Maret, $A$ et al. Agenesis of the human pancreas due to decreased half-life of insulin promoter factor 1. J Clin Endocrinol Metab. 2003;88:4398-406.

39. Wildin RS, Smyk-Pearson S, Filipovich AH. Clinical and molecular features of the immunodysregulation, poliendocrinopathy, enteropathy, $X$ linked (IPEX) syndrome. J Med Genet. 2002;39:537-45.

40. Delepine M, Nicolino M, Barrett T, Golamaully M, Lathrop GM, Julier C. EIF2AK3, encoding translation iniciation factor 2-a kinase 3 , is mutated in patients with Wolcott-Rallison syndrome. Nat Genet. 2000;25:406-9.

41. Sellick GS, Barker KT, Scolte-Dijkstra I, Fleischmann C, Coleman RJ, Garrett C, et al. Mutations in PTF1A cause pancreatic and cerebellar agenesis. Nat Genet. 2004;36:1301-5. 
42. Zung A, Glaser B, Nimri R, Zadik Z. Glibenclamide treatment in permanent neonatal diabetes due to an activating mutation in Kir6.2. J Clin Endocrinol Metab. 2004;89:5504-7.

43. Tonini G, Bizzarri C, Bonfanti R, Vanelli M, Cerutti F, Faleschini $E$, et al. Sulfonylurea treatment outweights insulin therapy in short-term metabolic control of patients with permanent neonatal diabetes mellitus due to activating mutations of the KCNJ11 (Kir6.2) gene. Diabetologia. 2006;49:2210-3.

44. Pearson ER, Flechtner I, Njolstad PR, Malecki MT, Flanagan SE, Larkin B, et al., the Neoantal Diabetes International Collaborative Group. Switching from insulin to oral sulfonylreas in patients with diabetes due to Kir6.2 mutations. N Engl J Med. 2006;355:467-77.

45. Gurgel LC, Crispim F, Noffs MH, Belzunces E, Rahal MA, Moisés RS. Sulfonylrea treatment in permanent neonatal diabetes due to G53D mutation in the KCNJ11 gene: improvement in metabolic control and neurological function. Diabetes Care. 2007;30:e108.

46. Gloyn AL, Ellard S. Defining the genetic aetiology of monogenic diabetes can improve treatment. Expert Opin Pharmacother. 2006;7:1759-67.

\section{Endereço para correspondência}

Regina S. Moisés

Universidade Federal de São Paulo, Escola Paulista de Medicina, Disciplina de Endocrinologia

Rua Botucatu, 740, $2^{\circ}$ andar

04034-970 São Paulo, SP, Brasil,

E-mail: rmoises@unifesp.br 